Cahiers de recherches médiévales

\title{
Jules César dans les Politiques de Juste Lipse (1589)
}

\section{Alexandre Tarrête}

\section{(2) OpenEdition \\ Journals}

Édition électronique

URL : https://journals.openedition.org/crm/2568

DOI : $10.4000 / \mathrm{crm} .2568$

ISSN : 1955-2424

Éditeur

Honoré Champion

Édition imprimée

Date de publication : 30 juin 2007

Pagination : 111-125

ISSN : 1272-9752

\section{Référence électronique}

Alexandre Tarrête, « Jules César dans les Politiques de Juste Lipse (1589) », Cahiers de recherches médiévales [En ligne], 14 spécial | 2007, mis en ligne le 30 juin 2010, consulté le 15 décembre 2022. URL : http://journals.openedition.org/crm/2568; DOI : https://doi.org/10.4000/crm.2568 


\section{酷M}

\section{Jules César dans les Politiques de Juste Lipse (1589)}

Les Politiques ${ }^{1}$ de Juste Lipse s'inscrivent dans la tradition ancienne des «miroirs du Prince », déjà bien établie au Moyen $\mathrm{Age}^{2}$ et illustrée à nouveau par la Renaissance ${ }^{3}$. Pour renouveler le genre, Lipse fit le choix d'une technique originale : celle du centon, dont il explique le principe dans sa préface ${ }^{4}$. Il s'agit d'emprunter aux historiens et aux philosophes antiques des citations (en vers et en prose, en latin ou en grec) pour bâtir ensuite un texte qui exprime une pensée originale à travers les mots les plus incisifs des meilleurs auteurs. L'exercice permet à l'auteur de faire montre d'érudition et de virtuosité, mais le procédé se justifie surtout par ses avantages pédagogiques : car le lecteur sera plus facilement convaincu par les mots dorés empruntés aux plus grands auteurs (dont le nom s'affiche en manchettes), et les sentences ainsi détachées s'imprimeront plus durablement dans sa mémoire.

Le recours à la figure de César dans ce traité doit se comprendre dans le cadre rhétorique ainsi défini : en voulant s'adresser avant tout aux rois et aux princes d'une Europe alors mise à feu et à sang par les divisions religieuses, Lipse entend proposer à ses lecteurs des modèles d'autorité et de prudence qui puissent les aider à affermir leur pouvoir et à rétablir la paix dans leurs États. Si le personnage de César peut constituer l'un de ces modèles, c'est au prix d'une sélection des traits retenus qui laisse de côté ses facettes négatives ou ambiguës. Pourtant, dans un livre précé-

\footnotetext{
${ }^{1}$ Politicorum sive civilis doctrince libri sex. Qui ad Principatum maxime spectant, Leyde, Plantin-Raphelengius, 1589 , in- $4^{\circ}$. Nous renvoyons dans les pages qui suivent à l'édition critique de J. Waszink : Justus Lipsius, Politica, Assen, Royal Van Gorcum, 2004 (qui suit le texte paru à Anvers en 1599). Cette édition remarquable, avec traduction anglaise, est précédée d'une introduction très complète de 250 pages. On y regrette seulement l'absence d'un index des auteurs-sources cités en manchettes, qui aurait complété utilement l'index des noms propres.

${ }^{2}$ Voir par exemple L. K. Born, «The Specula Principis of the Carolingian Renaissance», Revue belge de Philologie et d'Histoire, XII, 1933, p. 583-612.

${ }^{3}$ Le genre est illustré en particulier par Érasme dans l'Institutio principis christiani, adressée en 1516 à Charles de Bourgogne, ou par Budé, qui présente en 1519 à François $\mathrm{I}^{\mathrm{er}}$ le texte qui sera publié après sa mort, en 1547, sous le titre d'Institution du Prince. Voir La Formazione del Principe in Europa dal Quattrocento al Seicento, éd. P. Carile, Rome, Aracne, 2004 ; sur la période qui suit, voir I. Flandrois, L'Institution du Prince au début du XVII siècle, Paris, PUF, 1992.

${ }^{4}$ Voir la préface intitulée De Consilio et Forma nostri Operis (éd. cit., p. 230); sur la technique du centon dans les Politiques, voir J. Lafond, «Le centon et son usage dans la littérature morale et politique », L'Automne de la Renaissance (1580-1630), dir. J. Lafond et A. Stegmann, Paris, Vrin, 1981, p. 117-125 ; J. Waszink, «Instances of classical citations in the Politica of Justus Lipsius: their use and purposes », Humanistica Lovaniensia, XLVI, 1997, p. 240-257.
}

Cahiers de Recherches Médiévales, 14spé, 2007 
dent, Lipse avait présenté une image fort sombre du personnage ${ }^{5}$ : dans le De Constantia en effet, César apparaissait comme un tyran sanguinaire et une calamité pour son peuple ${ }^{6}$. L'image très positive de César dans les Politiques peut alors surprendre ; mais elle ne reflète pas tant le sentiment intime de l'auteur que la stratégie rhétorique qu'il s'est fixée pour ce nouveau livre. Il n'est donc pas inutile, avant d'étudier les différentes apparitions du personnage de César dans les Politiques, de présenter rapidement le contenu et la destination de ce traité et d'esquisser ce que fut sa réception.

\section{Les Politiques : présentation, destination et diffusion}

Juste Lipse enseigne l'histoire et le droit à l'université de Leyde de 1578 à 1591. C'est là qu'il rédige et publie les Politiques, en 1589. Le traité se compose de six livres ${ }^{7}$. Le premier présente les vertus morales et intellectuelles que le Prince doit cultiver, au nombre desquelles la piété, la probité, la prudence, la science. Le second livre traite du meilleur gouvernement (la monarchie), et des vertus qui peuvent conforter le pouvoir : la justice, la clémence, la foi et la prudence. Les livres III et IV traitent en particulier de la prudence en matière religieuse puis en matière civile. Lipse prend ici certaines positions qui donnèrent matière à controverse : il préconise l'unicité de la religion dans l'État, autorise l'usage de la force pour y parvenir ; il autorise aussi le Prince à recourir à l'usage limité de la tromperie ou du crime (fraus), mais dans le seul but d'affermir un pouvoir dont la destination reste le bien du peuple (malgré les précautions prises, on a lu dans cette conception de la prudentia mixta une concession limitée au machiavélisme) $)^{8}$. Le livre $\mathrm{V}$ traite plus spécifiquement de la prudence militaire, et aborde les questions de la stratégie et de l'autorité sur le champ de bataille. Le livre VI traite de la guerre civile. Lipse la condamne très sévèrement et en examine les causes et les remèdes. Ce rapide survol montre combien, en dépit de la forme du centon, qui semble donner la part la plus belle aux citations d'auteurs classiques, les Politiques expriment en fait une pensée originale et engagée.

Contrairement aux autres «miroirs du Prince», les Politiques ne sont pas dédiés à un Prince précis mais sont offerts à tous les souverains d'Europe. Ils s'ouvrent

\footnotetext{
${ }^{5}$ Sur les variations de l'image de César dans l'ensemble de l'œuvre lipsienne, voir P. H. Schryvers, «La présence de César dans Juste Lipse», Présence de César, éd. R. Chevallier, Paris, Les Belles Lettres, 1985, p. 239-245.

${ }^{6}$ Dans le De Constantia (Anvers, Plantin, 1584), l'image de César est très négative: poignardé en plein Sénat et devant une statue de Pompée, il figure au nombre des tyrans victimes de la vengeance tardive mais éclatante de Dieu (II, 15); ses nombreux massacres sont dénoncés (II, 22), tout comme les impôts excessifs qu'il a levés (II, 23).

${ }^{7}$ Sur la pensée de Lipse dans les Politiques, voir G. Oestreich, Antiker Geist und moderner Staat bei Justus Lipsius (1547-1606), Göttingen, Vandenhoeck und Ruprecht, 1989; M. Sénellart, «Le stoïcisme dans la constitution de la pensée politique: les Politiques de J. Lipse (1589) », dans Le Stö̈cisme au XVI et au XVII siècle, dir. P.-F. Moreau, Paris, Albin Michel, 1999, p. 117-139 ; J. Lagrée, Juste Lipse. La Restauration du stö̈cisme, Paris, Vrin, 1994, p. 80-96.

${ }^{8}$ La traduction du livre IV par Simon Goulart a fait l'objet d'une édition séparée, annotée et préfacée par J. Lagrée : Les Politiques. Livre IV, Caen, Presses Universitaires de Caen, 1994.
} 
sur une adresse ainsi libellée: «Imperator, reges, principes» («à l'empereur, aux rois et aux princes»). Lipse en envoie effectivement des exemplaires aux différents princes d'Europe. Parmi les destinataires attestés figurent Maurice de Nassau, qui fut son élève à l'Université de Leyde et qui restera l'un des hommes forts des PaysBas dans cette période troublée ; l'empereur Rodolphe II de Habsbourg (qui règne de 1576 à 1612); le comte palatin Frédéric IV (1583-1610); Maurice de Hesse (qui règnera après la mort de son père Guillaume en 1592); Christian I ${ }^{\mathrm{er}}$ prince d'Anhalt ; Christian IV, roi de Danemark et de Norvège (1588-1648). Lipse envoie aussi son livre à ses collègues de l'Université, aux édiles locaux, et à un certain nombre d'humanistes européens, parmi lesquels Marnix de Sainte-Aldegonde, Franciscus Junius, Montaigne, Pierre de Brach, Isaac Casaubon, etc ${ }^{9}$.

Au-delà de ces envois ciblés, la diffusion des Politiques est très large en Europe, comme en témoigne le nombre des éditions et des traductions : une cinquantaine d'éditions du XVI ${ }^{\mathrm{e}}$ au XVIII ${ }^{\mathrm{e}}$ siècle (jusqu'en 1752). En français, deux traductions: celle de Charles Le Ber publiée à La Rochelle en 1590 et dédiée à Saint-Luc, gouverneur de Brouage ; celle de 1594, due à Simon Goulart, et publiée à Genève. Le traité est aussi traduit dès les années 1590 en néerlandais, en anglais, en polonais et en allemand, et en italien dans les années 1600. Le succès a dépassé les prévisions de l'auteur, qui désapprouvait les traductions de son œuvre en langue vernaculaire (au motif que les citations empruntées à des auteurs latins perdent une grande partie de leur force en quittant leur langue d'origine).

Cette diffusion massive témoigne d'une réception qui dépasse largement la destination première que son auteur donnait au livre : le livre est lu non seulement par les princes ou par leurs conseillers, mais par les plus instruits de leurs sujets. L'ouvrage connaît ainsi un grand et durable succès en Europe.

\section{Le contexte d'origine}

Né dans la région de Louvain, où il a fait ses études, Lipse a trouvé refuge à l'université de Leyde en $1578^{10}$. La région est en paix mais reste sous la menace de la guerre qui fait rage au sud. Lipse ressent très vivement les désordres liés aux guerres civiles qui déchirent l'Europe : il exprime sa douleur devant les malheurs de sa patrie dans le De Constantia (1584) ${ }^{11}$. Ce contexte troublé explique que la théorie politique qu'il élabore dans les Politiques soit guidée par la recherche des principes pouvant garantir un pouvoir fort et le retour de la paix : défense du régime monarchique, principe d'unité religieuse dans l'État, concession limitée au machiavélisme destinée à affermir le pouvoir légitime du Prince, intérêt pour les matières militaires (la force étant l'un des fondements de la stabilité d'un régime) et prise en compte de

\footnotetext{
${ }^{9}$ Voir J. Waszink, Politica, éd. cit., préface, p. 114-115.

${ }^{10}$ On trouvera une présentation de l'œuvre et de la vie de Lipse due à C. Mouchel et M. Magnien dans les Centuria Latina. Mélanges offerts à J. Chomarat, éd. C. Nativel, Genève, Droz, 1997, p. 505-513. Signalons aussi le catalogue des livres de Lipse conservés à la Bibliothèque royale de Belgique, précédé d'une riche introduction: R. Adam et $\mathrm{M}$. de Schepper, Bibliotheca Lipsiana Bruxellensis, Bruxelles, Brepols/Musée de la Maison d'Érasme, 2006.

${ }^{11}$ Sur le De Constantia, voir par ex. J. Papy, «Lipsius' (Neo-)Stoicism : constancy between Christian faith and Stoic virtue », Grotiana, 22-23, 2001-2002, p. 47-71.
} 
l'opinion publique (la vertu du Prince sert avant tout à assurer sa popularité et donc son autorité).

Au milieu des années 1580, Lipse accueille favorablement l'arrivée du comte de Leicester, élu comme gouverneur en 1586 par les États généraux des Pays-Bas. Lipse voit en lui un homme capable de restaurer un pouvoir fort. Il espère même un temps jouer auprès de lui un rôle de conseiller et lui donne en particulier une leçon sur le De Agricola de Tacite. Mais le séjour de Leicester tourne court et il doit quitter le pays dès 1587 . La perspective d'un régime monarchique s'éloigne. Lipse dénonce dans sa correspondance la corruption des députés des États et la confusion d'un régime où les corps intermédiaires se disputent le pouvoir ${ }^{12}$.

La publication des Politiques déclenche aussi une controverse en matière religieuse. Selon Lipse, le pluralisme religieux affaiblit l'État et constitue une menace de guerre civile. Mais l'un de ses lecteurs, Dirk Coornhert, l'accuse de mettre en cause la liberté de conscience, principe auquel les habitants des Pays-Bas sont très attachés. S'ensuit un échange de libelles et une querelle qui affecte beaucoup Lipse, et qui a sans doute précipité son départ. Il ne semble pas pourtant que sa position à l'Université de Leyde en ait été affaiblie. Une autre contestation vient des lecteurs catholiques. La première édition des Politiques fut mise à l'Index. Les points de friction étaient nombreux : l'idée que la religion extérieure puisse différer de la religion intérieure, une condamnation jugée trop timide de Machiavel, le primat du politique sur le religieux, l'importance trop grande accordée au destin. Une fois retourné à Louvain, en terre catholique, Lipse a publié en 1596 une version révisée de son texte et a obtenu qu'il ne figure plus sur la liste des livres interdits.

Ce rapide survol des idées politiques de Lipse permet de comprendre son intérêt pour le personnage de Jules César, qui dessine la figure d'un Prince autoritaire, habile dans la paix comme à la guerre, capable de pacifier une Europe déchirée par les guerres de Religion. Dans les Politiques, il n'est pas le seul modèle proposé à l'imitation des Princes: Alexandre et Auguste, Pompée, Hannibal ou Marius sont également cités. Mais César occupe une place de choix : il apparaît dès la préface, et figure ensuite à des endroits stratégiques du texte (totalisant une vingtaine d'occurrences). Nous allons à présent nous attacher à dégager les différents rôles qui lui sont donnés dans les Politiques.

\section{Un traité placé sous le patronage de César}

La dédicace des Politiques à l'empereur, aux rois et aux princes européens est relativement brève, et elle contient une seule citation d'auteur ancien : c'est une citation de Lucain qui donne précisément la parole à César, à qui Lipse a ainsi confié le rôle de capter la bienveillance de ses lecteurs princiers. Ces quelques mots expriment la relation du Prince à ses sujets, qui dépendent entièrement de sa protection :

Atque ut a sole in subjecto hoc orbe lux aut tenebrae : sic a Principe apud subditos prava pleraque aut recta. Caesar ille non animose magis quam vere dixit:

${ }^{12}$ J. Waszink, Politica, éd. cit., préface, p. 25 et suiv. 
Procerum motus hac cuncta sequuntur.

Humanum paucis vivit genus ${ }^{13}$.

Peu importe que ces mots, chez Lucain, servent de remontrance à un soldat mutin, auquel César fait remarquer son insignifiance, en lui disant avec mépris qu'il trouvera sans peine des remplaçants aux soldats qui lui seront infidèles. Coupée de son contexte d'origine, la citation affirme au seuil du traité des Politiques le principe monarchique dans toute sa force: des décisions et des passions d'un seul dépend le sort de tous ses sujets. Le commentaire qui suit dégage alors la nécessité de conseiller le Prince pour s'assurer qu'il prendra des décisions salutaires pour tous :

Ergo eximia hac ratio de republica bene merendi, de Principe bene mereri : id est, ducere eum et dirigere ad metam illam Publici boni ${ }^{14}$.

César apporte ainsi sa caution au projet même d'un «Miroir du Prince» comme les Politiques : puisque tout dépend des grands, il importe qu'ils écoutent les conseils de l'histoire et de la philosophie, et ils pourront les trouver rassemblés dans le livre de Lipse. Même si la citation est détachée et réinterprétée en un sens neuf, son contexte original est toujours présent, par réminiscence implicite: dans la Pharsale, César s'adresse avec vigueur à un soldat mutin. Il affirme alors une autorité capable de ramener l'ordre dans le camp, et de mettre fin à la sédition. Les Politiques sont ainsi placés sous la figure emblématique de César pacifiant la révolte par sa présence et son verbe. À son instar, le Prince dont les Politiques font le portrait idéal est un pacificateur, un homme d'autorité, conscient aussi de son pouvoir et de ses devoirs envers son peuple.

Au fil des citations qui convoquent ensuite son image, César apparaît soit comme un expert en matière politique ou militaire, soit comme un exemple de vertu. Il faut toutefois noter qu'en tant qu'historien, César est rarement mis à contribution dans les Politiques. Lipse le considère manifestement davantage comme un homme d'action que comme un écrivain.

\section{Le jugement de Lipse sur César historien}

On est frappé par la présence très modeste de César parmi les historiens cités dans les Politiques. J'ai relevé seulement six citations directes de ses œuvres : trois viennent du De Bello gallico, trois du De Bello civile. On peut y ajouter deux citations tirées du De Bello alexandrino (un texte qui fait partie du corpus césarien et probablement rédigé sur son ordre) : Lipse, dans les manchettes des Politiques, indexe ce livre comme s'il s'agissait d'un texte de César et formant la suite du $D e$ Bello civile. Au total, cette poignée d'occurrences pèse bien peu en regard du nom-

${ }^{13}$ J. Lipsius, Politica, éd. J. Waszink, op. cit., p. 225-227 (les italiques qui détachent les citations sont de Lipse). Ma traduction : «Et de même que ce monde-ci reçoit du soleil la lumière ou les ténèbres, de même les sujets reçoivent tout du Prince, le bon comme le mauvais. Le grand César déclara avec autant d'énergie que de vérité : Toutes ces choses sont les suites des mouvements des grands; la vie du genre humain tout entier tient en celle de peu d'hommes » (Lucain, BC, 5.342-343).

${ }^{14} \mathrm{Ibid}$. ( C'est pourquoi la façon la plus remarquable de servir l'intérêt commun est de servir le Prince ; c'est-à-dire de le guider et de le diriger vers ce grand but qu'est le bien commun »). 
bre impressionnant des citations empruntées à Tacite, le plus sollicité de tous : Jan Waszink en dénombre pas moins de $528^{15}$.

Dans sa préface sur le projet et la forme de son œuvre, Lipse explique pourtant qu'il a glané des sentences chez les écrivains antiques en privilégiant les historiens, qui sont «la source même de la prudence civile ${ }^{16}$ ». On pourrait donc s'attendre à trouver César en bonne place aux côtés des autres historiens latins cités dans l'ouvrage. Il figure bien dans la liste des sources au début du livre, mais tout à la fin ${ }^{17}$. Tacite, que Lipse avait édité en 1574, est distingué avant tous les autres. Ensuite, une première liste assez brève présente les auteurs qu'il a utilisés le plus souvent et le plus volontiers : elle comprend surtout des historiens et des philosophes: Salluste, Tite-Live, Sénèque, Cicéron, Quinte Curce, Pline le Jeune, Végèce (auteur de l'Epitoma res militaris), Aristote, Thucydide, Platon, Xénophon. Une seconde liste, plus longue, rassemble les auteurs utilisés plus rarement ; César y figure, perdu parmi 70 sources latines et 35 sources grecques. Il ne fait donc pas partie des historiens du premier cercle. Il est possible que le jugement très favorable de Cicéron sur le style de César ait joué en défaveur de ce dernier auprès de Lipse, qui fit assez tôt profession d'anti-cicéronianisme et préféra imiter Sénèque ou Tacite ${ }^{18}$. Il est possible aussi que Lipse, qui veut donner César en modèle à des Princes et non à des clercs ou à des érudits, choisisse de ne pas insister sur son activité d'écrivain. Un parti pris différent s'observe à la même époque chez l'un des correspondants les plus fameux de Lipse : Montaigne ${ }^{19}$.

Lipse exprime son jugement mitigé sur l'historien César dans les Nota qu'il publie dès 1589 comme un complément à son traité. Parmi les historiens latins, dans un ordre qui est donné explicitement comme un classement, César vient après Tacite, Salluste, Tite-Live et même Quinte Curce, devançant seulement le dernier de la liste : Ammien Marcellin. Le jugement détaillé que Lipse donne de César permet de comprendre ce qui motive cette relégation :

C. Casar res suas ipse digessit: composite sane, et pari modestia ac libertate. In stilo sunt qua culpem, sine ejus tamen culpa. Nam malam aliquam et alienam manum accessisse, satis mihi liquet. Prudentiam non e verbis ejus hauries, sed e factis :

${ }^{15}$ Politica, éd. cit., préface, p. 163.

${ }^{16}$ Politica, éd. cit., p. 232.

${ }^{17}$ Ibid., p. 254-258.

${ }^{18}$ Voir M. W. Croll, «Juste Lipse et le mouvement anticicéronien à la fin du XVI ${ }^{\mathrm{e}}$ siècle et au début du XVII ${ }^{\mathrm{e}}$ siècle», repris dans Style, rhetoric and rhythm, éd. J. M. Patrick et R. O. Evans, Princeton, Princeton University Press, 1966, p. 7-45 ; C. Mouchel, «Les rhétoriques post-tridentines (1570-1600)», Histoire de la rhétorique dans l'Europe moderne (14501950), dir. M. Fumaroli, Paris, PUF, 1999, p. 431-497.

${ }^{19}$ Sur l'admiration portée à César écrivain, voir F. Charpentier et B. Boudou, «La figure de Jules César dans le Discours de La Boétie et dans les Essais de Montaigne ", Cahiers de Recherches Médiévales, 13, 2006, p. 187-188; B. Méniel, "César écrivain, d'après les lecteurs de la Renaissance », ibid., p. 219-220. 
et maxime Militarem. Commentarii enim sunt, et nihil pollicentur preter nudam simplicemque narrationem ${ }^{20}$.

Lipse considère les livres de César comme de simples «Commentaires », c'est-à-dire des journaux de campagne, il en juge le style nu, simple, un peu fautif parfois ; il les oppose implicitement à la grande narration historique. Il considère donc César moins comme un écrivain que comme un homme d'action et avant tout comme un général. César est plus un modèle de prudence par ses actes que par ses mots ${ }^{21}$. Notons aussi, au passage, que Lipse estime ici que César est une référence plus incontestable en matière de prudence militaire qu'en matière de prudence civile - même si, dans les Politiques, ces deux domaines sont tour à tour l'occasion de mobiliser son exemple.

\section{César, expert en matières militaires}

Dans les matières militaires, tout d'abord, César apparaît dans les Politiques comme une autorité incontestée. On retrouvera d'ailleurs cette image du chef de guerre exemplaire dans des œuvres que Lipse écrira plus tard : le De Militia romana (Louvain, 1594, dédié à Philippe III) ou le De Magnitudine romana (Louvain, 1598, dédié à Albert d'Autriche) ${ }^{22}$. La plupart des références au César imperator trouvent naturellement leur place dans le livre $\mathrm{V}$ des Politiques, consacré à la prudence militaire $^{23}$. Le champ de bataille est par excellence le terrain où la Fortune manifeste sa toute puissance sur la destinée des hommes et des États. Mais son importance peut être équilibrée par la prudence. Dans le chapitre 16, consacré à l'importance de la stratégie, César est sollicité d'une part pour attester l'importance de la Fortune dans les succès militaires et d'autre part pour confirmer l'importance de la stratégie :

\footnotetext{
${ }^{20}$ "César a mis en ordre ses propres actions: avec une belle ordonnance, et autant de modestie que de liberté. Il y a des choses que je blâmerais dans son style, sans que ce soit vraiment des fautes de sa part. Mais il est assez clair pour moi qu'une main étrangère et maladroite a ajouté à son texte. Tu ne tireras pas la prudence de ses mots, mais de ses actes : et surtout en matière militaire. Ce sont en effet des Commentaires, et ils ne sont en rien polis davantage qu'une narration simple et nue » (Ad libros politicorum breves notce, I, 9, dans les Politica, éd. cit., p. 734). Ce jugement est textuellement repris par La Popelinière, qui ne mentionne pas sa source: voir L'Histoire des Histoires [...], Paris, M. Orry, 1599, I, p. 304 (passage cité par B. Méniel, «César écrivain d'après les lecteurs de la Renaissance », art. cit., n. 16 p. 209).

${ }^{21}$ La prudentia renvoie d'abord à une vertu morale, qui peut d'ailleurs s'exprimer dans la forme (voir B. Méniel, ibid., p. 211-217), mais elle peut aussi désigner une qualité purement stylistique : J.-C. Scaliger, par exemple, en fait l'une des vertus cardinales du poète (Voir les Poetices libri septem, Lyon, 1561, Stuttgart, F. Frommann, 1964, III, 26, p. 113) - je remercie Virginie Leroux de m'avoir indiqué ce texte.

${ }^{22}$ Voir P. H. Schryvers, art. cit., p. 240.

${ }^{23}$ Peu avant sa mort, Lipse travaillait à un recueil manuscrit d'exempla sur la prudence militaire, pour prolonger et compléter ce chapitre (voir J. Papy, « An unpublished dialogue by J. Lipsius on military prudence and the causes of war: the Monita et exempla politica de re militari (1605)», BHR, LXV, 2003, 1, p. 135-148). César y est mis à contribution pour illustrer les motivations financières des conflits : il a traversé la Manche dans l'espoir de trouver, d'après Suétone, des perles et, d'après Cicéron, de l'argent (loc. cit. p. 145).
} 
Multum cum in omnibus rebus, tum in re militari potest fortuna, Tamen profecto Consilii quoque magnae et validae hic partes sunt ${ }^{24}$.

La puissance de la Fortune, dont César a fait l'expérience sur les champs de bataille, ne doit pas dissuader de cultiver la prudence et d'élaborer une stratégie. Comme chez Machiavel, la prudence est vue comme une force capable de neutraliser parfois la Fortune et sur laquelle il faut s'appuyer, tout en sachant que le succès n'est jamais certain. César intervient aussi pour souligner l'utilité et la dignité de la stratégie, qui ne diminue pas le courage et concourt également à la victoire :

Ideo Caesar definiit, Non minus esse Imperatoris consilio superare, quam gladio ${ }^{25}$.

César sert ici de caution à la réflexion théorique sur l'art militaire dont Lipse se réclame dans le livre $\mathrm{V}$ : un grand conquérant comme lui n'a pas dédaigné la réflexion et la stratégie, et tout Prince devrait s'inspirer de son exemple.

À côté de ces principes assez généraux, on trouve aussi des recommandations techniques plus précises. César fournit des préceptes sur la discipline militaire, nécessaire à l'obéissance : elle nécessite une stricte limitation des gratifications et des largesses, qui sapent l'endurance des troupes ${ }^{26}$. S'il est arrivé à César, en raison de son charisme hors du commun, d'autoriser un certain luxe à ses soldats, il ne doit pas être pris comme modèle sur ce point : ses légionnaires équipés d'armes précieuses, serties d'or et d'argent, et parfois même couverts de parfums précieux pour aller au combat ne sont cités que comme une exception curieuse qui déroge aux principes de l'art militaire ${ }^{27}$. Telle est au fond l'ambiguïté de César : souvent exemplaire, il est parfois trop atypique pour être proposé à l'imitation des chefs de guerre ordinaires.

Il n'en reste pas moins qu'il a incarné le chef de guerre idéal. Sa vigilance et sa concentration sont citées en exemple, car le sort d'une bataille peut basculer sur de tout petits détails ${ }^{28}$. Sa finesse lui permet de saisir le temps le plus propice aux pourparlers : c'est celui où les deux camps semblent de force égale, car alors aucun ne sera tenté de pousser son avantage ${ }^{29}$. César brille enfin par ses qualités d'homme de terrain. Il n'est pas seulement un stratège ou un théoricien mais aussi un général renommé pour son courage et son goût de l'engagement physique. Il est un exemple à suivre pour tous les chefs qui ne doivent pas hésiter, lorsque le moral des soldats est ébranlé, à venir en personne les exhorter :

\footnotetext{
${ }^{24}$ Politica, V, 16, éd. cit. p. 620 («la Fortune est puissante dans toutes les affaires, et aussi dans les affaires militaires $[B G, 6.30 .2]$. [...] Pourtant le rôle de la stratégie est aussi grand et important $»$.

${ }^{25}$ Ibid. («Ainsi César a établi, qu'il n'est pas moins digne d'un chef de vaincre par la stratégie que par le glaive $[B C, 1.72 .3] »)$.

${ }^{26}$ Voir Politica, éd. cit., V, 13, p. 604 [Bell. Alex. 48].

${ }^{27}$ Voir Politica, V, 13, p. 600 [Suét. Jul. 67.2].

${ }^{28}$ Voir Politica, V, 16, p. 624 [Caes. $\left.B C, 1.21 .1\right]$.

${ }^{29}$ Voir Politica, V, 19, p. 656 [Caes. BC, 3.10.7].
} 
Audi poetam de Caesare :

Ipse manu subicit gladios, ac tela ministrat,

Promovet ipse acies, impellit terga suorum,

Verbere conversa cessantes excitat hasta ${ }^{30}$.

La citation, empruntée à Lucain, est habilement tronquée par Lipse, afin de laver l'image de César de ses aspects négatifs. Il conserve les trois vers (574 et 576-577) qui insistent sur le courage de César, combattant en première ligne avec ses soldats, mais il ôte le vers intermédiaire (adversosque jubet ferro contundere vultus ${ }^{31}$ ) qui trahissait sa cruauté et sa frénésie meurtrière. Le César de Lucain est en effet un personnage inquiétant, tenu pour responsable du sang versé dans la guerre civile: Lipse, pour des motifs pédagogiques, efface ces zones d'ombre pour ne conserver que la leçon de courage donnée aux soldats. La technique du centon permet et appelle ces détournements de sens.

\section{César, modèle du chef politique}

César n'est pas seulement un général ou un expert en matière militaire : il est aussi celui qui a mis fin aux guerres civiles et qui a présidé pendant plusieurs années aux destinées de la République romaine. Il dispose à l'évidence d'un savoir précieux sur les moyens de conquérir et de conserver le pouvoir et sur les difficultés auxquelles se heurtent tous ceux qui ont à diriger un Etat avec prudence. Les thèmes développés dans le cadre strictement militaire du livre V sont aisés à transposer dans une réflexion sur l'action politique en général. Les qualités de commandement ou de décision propres à l'homme de guerre trouvent naturellement à s'appliquer dans le champ politique, surtout en temps de guerre civile. Par exemple, dans le chapitre III, 8 (sur l'importance de la réflexion et des conseils), une phrase du De Bello gallico attire l'attention sur l'imprudence de celui qui délibère dans la précipitation :

Mature deliberet. Timet, atque eum deficere omnia videntur, qui in ipso negotio consilium capere cogitur ${ }^{32}$.

La citation s'appliquait à l'origine à dénoncer l'erreur tactique commise par le centurion Titurius qui, à cause de son imprévoyance, ne savait pas comment manœuvrer alors que l'armée romaine était tombée dans une embuscade. Tirée d'un contexte militaire et anecdotique, cette maxime voit sa portée élargie par Lipse qui l'isole et lui donne une valeur générale : un chef doit délibérer à loisir, et prendre conseil bien avant l'action.

\footnotetext{
${ }^{30}$ Politica, V, 16, p. 638 («Écoute ce que dit le poète de César : Lui même donne de sa propre main les glaives, et distribue les traits. [...] Il fait lui-même avancer la ligne de bataille, pousse le dos de ses soldats, et excite ceux qui tardent d'un coup de sa lance renversée» [Lucain $B C$, VII, v. 574 et 576-577]).

${ }^{31}$ «Et il ordonne de meurtrir par le fer le visage des adversaires » [Ibid., v. 575].

${ }^{32}$ Politica, III, 8, p. 366 ("Qu'il délibère mûrement. Il délibère dans la crainte, et toutes choses semblent lui faire défaut, celui qui est forcé de délibérer en pleine action [Cés., $B G$, 5.33]»).
} 
Comme en matière militaire, César est consulté à nouveau en expert, mais cette fois sur les fondements de l'autorité civile. Dion Cassius lui attribue un propos qui dévoile le double fondement du pouvoir: la force armée et l'argent ${ }^{33}$. Ailleurs, César délivre un conseil sur la perception des impôts : il est important de surveiller les collecteurs pour qu'ils n'abusent pas du peuple, au risque de faire encourir l'impopularité au Prince qu'ils prétendent servir ${ }^{34}$. Enfin, César intervient pour attester le rôle clé de la vertu du chef dans la constitution de son autorité : les qualités de modération et de clémence (souvent associées à César) sont des vertus, mais aussi et surtout des choix réalistes qui doivent contribuer à conforter le pouvoir en s'assurant la bienveillance du peuple ${ }^{35}$.

César est à nouveau sollicité pour témoigner de la toute puissance de la fortune, qui tour à tour favorise et précipite la chute de ceux qu'elle avait un temps comblés de ses dons ${ }^{36}$. Le thème prend ici toute sa résonance: il ne s'agit plus seulement de la Fortune des champs de bataille, divinité erratique et contingente, mais du Destin, qui exprime pour un stoïcien comme Lipse la Providence et l'ordre du monde. La fortune, même si elle ne se maîtrise pas, reste indispensable à celui qui commande. Elle conforte l'autorité de celui qu'elle favorise ${ }^{37}$. Le personnage de César est traditionnellement lié avec les thèmes du hasard et de la fortune, en grande partie à cause de l'épisode emblématique du franchissement du Rubicon. Ce topos est évoqué sous une forme allusive, dans un passage qui traite de la nécessité de conclure des alliances sans pouvoir à l'avance être certain de la victoire de ses alliés. Lorsque deux voisins entrent en guerre l'un avec l'autre, il faut nécessairement choisir de s'engager avec l'un ou avec l'autre, et ce choix ne peut se faire sans risque :

Cum Caesare igitur Iacienda tunc alea : et alterutri se ajungendum ${ }^{38}$.

Lipse ne reprend pas les critiques qui avaient pu être formulées contre César pour avoir transgressé les lois de Rome et mis le sort de sa Patrie en danger pour des ambitions personnelles. En effet, le contexte est ici celui d'un conflit déclenché par des alliés et dans lequel le Prince est comme forcé de s'engager pour ne pas mécontenter à la fois ses deux puissants voisins. La responsabilité du conflit incombe ici à autrui. César est simplement celui qui sait prendre des risques lorsqu'ils sont inévitables.

\section{Au-delà des topoi}

Toutes les apparitions de César que nous avons recensées jusqu'à présent correspondent peu ou prou à la tradition positive ou négative attachée au personnage depuis l'Antiquité : César est lié au thème de la toute puissance de la Fortune qui a favorisé ses succès militaires et politiques, avant de l'abandonner à ses assassins ; il

\footnotetext{
${ }^{33}$ Politica, IV, 7, p. 414 [Dion Cassius 42.49.4].

${ }^{34}$ Politica, IV, 11, p. 476 [Caes. BC, 3.32.4].

${ }^{35}$ Politica, IV, 8, p. 420 [Dion Cassius, 46.16.3].

${ }^{36}$ Politica, IV, 9, p. 442 (Bell. Alex. 25.4).

${ }^{37}$ Politica, IV, 9, p. 444 [César, $B G, 7.30 .3$ ].

${ }^{38}$ Politica, IV, 9, p. 440 («Ainsi, avec César, les dés doivent alors être jetés [Suet. Jul. 33.1] : et il faut s'allier avec l'un des deux »).
} 
est loué pour ses qualités de chef de guerre (vitesse d'exécution, courage, autorité, charisme). Lipse fait fonds sur ces topoi qui lui sont utiles dans son projet rhétorique et pédagogique. Mais les leçons qu'il dispense dans les Politiques ne sont pas toujours topiques ou traditionnelles. Et il est intéressant de constater que le personnage de César est également utilisé lorsqu'il s'agit d'illustrer les points les plus novateurs de la pensée politique de Lipse.

Le livre VI des Politiques analyse la spécificité de la guerre civile, ses causes et ses remèdes. La guerre civile est plus grave que les guerres ordinaires entre peuples parce qu'elle sape les fondements mêmes du lien social. Elle met en péril l'ordre de la société mais aussi l'ordre même des armées. Lipse exploite ici une facette plus sombre du personnage de César: en prenant le risque de déclencher une guerre civile contre Pompée, il s'est engagé sur un chemin périlleux où la perte des repères moraux et politiques au sein même de son armée finit par menacer son autorité sur ses propres soldats. Dans une guerre illégitime, lorsque le chef est lui-même coupable d'une transgression, son autorité ne reste pas longtemps incontestée. Au seuil du livre VI, consacré à la guerre civile, Lipse place une évocation du désarroi des armées de César plongées dans une guerre illégale et criminelle. Il fait appel à Lucain qui présente les armées de César en pleine déliquescence. La guerre civile, en effet, rompt les liens de l'honneur et la fidélité qui unissent les soldats à leurs chefs. De cette crise morale témoigne la mutinerie des soldats révoltés contre César : lassés de guerroyer, ils refusent de passer en Grèce :

Rheni mihi Casar in undis

Dux erat, hic socius. Facinus quos inquinat, aquat ${ }^{39}$.

Cette harangue montre les dangers inquiétants de la guerre civile, qui menace directement le pouvoir militaire. La reprise en main de César n'est sans doute que plus urgente. Chez Lucain, il intervient quelques vers plus loin pour rétablir l'ordre. Dans la suite du livre VI des Politiques également, César ne tarde pas à reparaitre comme une figure charismatique capable de ramener la paix.

Le chapitre 4 examine si le Prince doit admonester lui-même le peuple rebelle. En général, non: il faut qu'il réserve son autorité pour de plus importants remèdes; mais par exception, oui : il peut parfois trouver le succès dans l'audace. Face à la rébellion, le Prince doit parfois payer de sa personne. César incarne cette prise de risque couronnée de succès. Face au soldat mutin qui a commencé à haranguer ses camarades en l'absence du chef, ce dernier surgit par surprise, et son courage fait forte impression sur les révoltés :

Fecit magnus Caesar, et

- stetit aggere fulti

\footnotetext{
${ }^{39}$ Politica, VI, 1, p. 668 («Sur les rives du Rhin, César était mon général; ici il n’est que mon compagnon. Le crime rend égaux ceux qu'il souille [Lucain, BC, 5.289-290]»).
} 
Cespitis, intrepidus vultu, meruitque timeri Nil metuens ${ }^{40}$.

L'exemple est d'ailleurs suivi par un exemple similaire mettant en scène Auguste, et pris chez Tacite (Hist. I. 21). Dans ces exemples - que Lipse présente comme des exceptions et non comme des préceptes - se laisse lire son désir de voir surgir dans les Flandres, aux Pays-Bas et en France peut-être des princes charismatiques qui pourront mettre un terme aux divisions sanglantes des guerres civiles.

Face aux défis des guerres civiles en Europe, il ne faut rien négliger pour renforcer le pouvoir des Princes, qui seul peut garantir la paix. C'est dans ce cadre que Lipse réfléchit à une réplique à la pensée de Machiavel, alors en pleine diffusion en Europe, et souvent dénoncée pour son immoralisme. Il choisit d'autoriser lui aussi le Prince à des actes contraires à la morale commune, mais de manière exceptionnelle et sous des conditions très restrictives. Dans le chapitre 14 du livre IV, Lipse avance l'idée que le Prince peut parfois commettre un crime pour conforter son pouvoir, mais à plusieurs conditions : que ce crime soit nécessaire, qu'il soit la seule voie possible, et qu'il serve à conforter un pouvoir que le Prince exerce en général pour le bien du peuple. Le recours au crime (fraus) est donc exceptionnel et il vaut mieux que son usage reste secret. Il s'agit pour Lipse de faire la part du feu face à Machiavel et aux théories de la raison d'État, qui prétendent affranchir le Prince de tout impératif moral.

C'est dans ce contexte que Lipse mobilise à nouveau la figure de César, dans un sens cette fois moins conforme aux éloges traditionnels. En s'appuyant sur une citation de Dion Cassius, il rappelle que le général romain éliminait de façon détournée ceux qu'il ne pouvait pas convaincre d'un crime suffisant, en s'arrangeant pour les faire assassiner sur le champ de bataille ${ }^{41}$. Si le grand César lui-même s'est permis de tels procédés, il semble y autoriser les autres princes. De telles actions, contraires à la morale ordinaire, ne sont pas contraires à la prudence, puisqu'elles aboutissent à fortifier le pouvoir. Celui-ci, chez Lipse, n'est pas amoral comme chez Machiavel, car il est exercé par un Prince qui recherche avant tout le Bien de ses sujets, en l'occurrence la fin des divisions et le retour de la paix. La fin justifie donc les moyens, et le Prince n'a pas à être exemplaire dans ses actions cachées. Il lui suffit de favoriser le retour à la concorde, et de veiller pour cela au renforcement de son propre pouvoir.

Pris malgré eux dans des guerres civiles qui les menacent, les simples citoyens ont d'ailleurs eux aussi la possibilité d'agir en faveur de ce retour à la paix. Au chapitre 6 du livre VI, Lipse se permet une digression : il cesse pour un moment de s'intéresser au Prince pour s'intéresser à ses sujets et, en particulier, aux hommes comme lui, qui souffrent des guerres sans y être impliqués directement. Ont-ils tort de ne pas s'engager dans les affrontements? Lipse pense que non, et il élabore une théorie très précise de l'abstention des citoyens neutres (la guerre civile étant par définition injuste, et confisquée par des intérêts particuliers). On trouve dans ces

${ }^{40}$ Politica, VI, 4, p. 684 («Le grand César le fit, et il se tint sur un petit rempart de gazon, avec un visage intrépide, et mérita d'être craint, puisqu'il ne craignait rien [Lucain, $B C$, 5.316-8]»).

${ }^{41}$ Politica, IV, 14, p. 526 [Dion Cassius, Hist. Rom. 43.13.2.]. 
mêmes années des réflexions convergentes chez Montaigne ou chez Du Vair sur la possibilité honorable de se retirer de la folie collective ${ }^{42}$.

Lipse trouve en César une caution de poids pour conforter sa position en la matière. Un premier exemple présente plutôt César dans une position belliciste: il cherche à retourner Asinius Pollion, ancien partisan de Pompée, et à l'entraîner dans son armée. Mais celui-ci refuse, et honorablement: il décide de se retirer de l'affrontement et de promettre par avance sa foi au vainqueur, quel qu'il soit ${ }^{43}$. Dans un deuxième temps cependant, c'est César lui-même qui donne aux citoyens neutres le conseil de rester hors du conflit. À la différence de Pompée qui considère comme des traîtres ceux qui ne s'engagent pas à ses côtés, César veut les considérer comme des alliés en puissance.

Vir optime salve, et probande magno illi Caesari : qui denuntiante Pompeio, pro hostibus se habiturum qui reipublica defuissent : ipse medios et neutrius partis, suorum sibi numero futuros pronunciavit ${ }^{44}$.

L'intérêt pour lui est double: il affirme à nouveau sa légendaire clémence, en pardonnant à ceux qui ont tardé à se rallier à lui. Mais il évite aussi d'embraser l'ensemble du pays, en cantonnant la guerre aux partisans déclarés, et en limitant ainsi sa durée et son extension. Si seulement les chefs des guerres de Religion pouvaient agir de la sorte! César apparaît donc ici comme un homme de paix, qui conseille l'abstention: posture quelque peu paradoxale, mais louable pour son pragmatisme et son pacifisme.

\section{César, figure du lecteur idéal d'un «miroir du Prince»}

La dernière figure de César que nous examinerons est encore plus inattendue: elle est celle d'un Prince qui aime s'instruire et qui n'aurait pas dédaigné d'ouvrir un livre comme les Politiques pour y trouver des avis et des préceptes fructueux. C'est le sens de la figure de César que l'on trouve au seuil du livre V, consacré à la prudence militaire. Dans un propos préfaciel qui repose sur l'argument topique de l'excusatio propter infirmitatem, Lipse se demande comment il pourra aborder un domaine - l'art militaire - sur lequel il a bien peu de lumières directes. Il trouve alors dans la figure de César et d'Alexandre des cautions bienveillantes. Ces Princes, en effet, n'ont pas répugné à lire les poètes, les historiens et les philosophes, et à y puiser des enseignements pratiques. Leur habileté à la guerre n'a donc pas l'expérience pour seule source. Or Lipse ne fait rien d'autre, à l'en croire, que rassembler les préceptes des écrivains antiques : il suppose alors implicitement, et non sans humour, que César lui-même aurait pris plaisir à lire ses Politiques.

${ }^{42}$ Voir Montaigne, Essais, éd. Villey/Saulnier, Paris, PUF, 1965, livre III, chap. 10 et Du Vair, De la Constance, Paris, L'Angelier, 1594, livre III.

${ }^{43}$ Politica, VI, 6, p. 700 [Vell. 2.86].

${ }^{44}$ Politica, VI, 7, p. 704 («Salut à toi, homme excellent, qui aurait l'assentiment de César : qui lorsque Pompée annonça qu'il considérerait tous ceux qui feraient défaut à la République comme ses ennemis, annonça quant à lui qu'il considèrerait ceux qui restaient neutres et ne prenaient pas parti pour l'instant comme ses partisans dans le futur [i. e. après sa victoire]» [Suet. Jul. 75.1]). 
Ego vero audebo. Nec mei tamen ingenii aut virium fiducia, sed eorum a quibus jamdiu mutuor et sumo. Quid enim hic meum? ordo aliquis et contextus fortasse : at verba sensusque mihi prceunt illi, quos in hac ipsa re (fidenter dicam) Annibal aliquis audire nihil abnuat, aut ipse Casar ${ }^{45}$.

Hannibal et César ne sont plus ici les sources de la sagesse, mais ses destinataires. Loin de devoir leur réussite à l'inspiration ou au génie, ils ont dû eux aussi étudier la prudence civile et militaire auprès de ceux qui les ont précédés. Ceux qui veulent leur ressembler doivent donc avant tout imiter leur humilité et leur soif d'apprendre.

Dans les Politiques, César apparaît souvent en position d'écoute, en raison des nombreux emprunts que Lipse fait aux Lettres que Salluste est censé lui avoir écrites. Les manchettes attribuent à Salluste ces deux textes, qui sont sans doute l'œuvre d'un rhéteur impérial ${ }^{46}$. Du reste, dans une édition de 1607 , soit un an après la mort de Lipse, le problème de l'authenticité de ces lettres était déjà débattu ${ }^{47}$. Ces lettres ouvertes conseillent à César toute une série de réformes politiques et judiciaires visant à l'égalité des citoyens et à la justice sociale, à la lutte contre les dépenses somptuaires des nobles ou des partisans, à la défense des libertés. Lipse fait une utilisation massive de ces deux épîtres: pas moins d'une cinquantaine d'emprunts disséminés dans les livres IV, V et VI des Politiques. Lipse y trouve des conseils pragmatiques pour un gouvernement juste, capable de mériter la considération du peuple et de conforter en retour l'autorité du Prince. La figure implicite de César n'est plus ici celle de l'expert ou du héros, mais celle du destinataire des conseils politiques, reflet des «princes, rois et empereurs» qui sont les lecteurs désignés du livre. Même s'il n'est plus sur le devant de la scène, il reste présent dans les marges du discours, comme celui qui écouta le premier les justes conseils de Salluste. Sa présence doit inciter les lecteurs princiers auxquels Lipse s'adresse à écouter attentivement ces conseils avec respect. D'exemple et de donneur de leçons politiques, César est devenu l'exemple d'un Prince à l'écoute de son conseiller. Cet habile retournement renforce encore l'identification du lecteur à César.

Les deux Lettres à César permettent, au cœur des Politiques, une mise en abyme du genre du Miroir du Prince. César harangué par Salluste symbolise la rela-

\footnotetext{
${ }^{45}$ Politica, V, 1, p. 534 ( $«$ J'oserai [aborder ce sujet]. Non par confiance en mon propre talent ou en mes propres forces, mais par confiance en ces hommes à qui je prends et $\mathrm{j}$ 'emprunte depuis longtemps. Car qu'est-ce qui est à moi ici ? L'ordre et le contexte peut-être. Mais pour les mots et les idées ces grands auteurs me guideront, eux qu'en ces matières Hannibal ou César eux-mêmes ne dédaignaient pas d'écouter »).

${ }^{46}$ Pseudo-Salluste, Lettres à César. Invectives, éd. et trad. A. Ernout, Paris, Les Belles Lettres, 1974 ; Pseudo-sallustian works, dans Sallust, éd. et trad. J. C. Rolfe, Londres/New York, Heinemann/Putnam, 1921.

${ }^{47}$ C. Crispi Sallustii Opera Omnia qua exstant, ex recognitione Jani Gruteri. Accedunt Castig. Annotat. Notee, ac Scholia Glareani, Popma, Aldi nepotis, Palmerii, Coleri, Rivii, Carrionis, Ursini, Dousa, Putschii, Francfort, J. Rhodius, 1607. Les notes de Dousa optent pour l'authenticité, que contestent celles de Carrio. Au vingtième siècle encore, la thèse de l'authenticité a été soutenue par M. Chouet (Les Lettres de Salluste à César, Paris, Les Belles Lettres, 1950).
} 
tion de lecture et d'écoute que Lipse souhaite établir avec les princes qui seront ses lecteurs. Au cœur du texte est ainsi figurée le mode de réception qu'il postule, et César fait figure de lecteur idéal.

Globalement, on peut dire que la présence de César dans les Politiques est significative, mais non écrasante. Une place de choix lui est réservée, en tête de l'ouvrage: il constitue le modèle immédiatement proposé à l'imitation des lecteurs princiers, et assure à ce titre la captatio benevolentice la plus efficace. Mais il faut bien reconnaître que dans l'ensemble du traité, en matière politique, la pensée de Lipse s'inspire bien davantage de Sénèque et de Tacite que de César. Mais sa figure est indispensable pour des raisons rhétoriques : sa célébrité et son charisme en font un modèle auquel les Princes qui liront le livre auront envie de ressembler. De plus, la situation historique qui fut la sienne le rapproche des hommes du seizième siècle : il est celui qui a su mettre fin au carnage des guerres civiles à Rome.

Le traité de Lipse est une œuvre hybride, qui tient à la fois du recueil de lieux communs et de la théorie politique la plus innovante. La figure de César se trouve prise dans cette ambivalence. Dans la mesure où Lipse renoue avec le genre médiéval du speculum principis, il hérite aussi des images les plus traditionnelles du personnage: sage, preux, expert en matières militaires, rompu à la science du gouvernement, favori de la Fortune jusqu'à sa mort tragique. Mais plus souterrainement, Lipse rompt avec la pensée politique traditionnelle sur bien des points, et le personnage de César l'accompagne aussi sur ces nouveaux terrains: il donne ses traits au Prince que Lipse appelle de ses voeux pour les pays d'Europe, farouchement opposé aux guerres civiles, encourageant les citoyens à cesser de combattre et à rester neutres, et prêt ensuite à pardonner aux ennemis d'hier pour rétablir la concorde. La stature du monarque absolu se détache déjà en filigrane de ce traité : le Prince rêvé par Lipse est autorisé à la «fraude légitime» mais seulement si celle-ci sert le bien commun; il est autoritaire et charismatique mais ouvert aux conseils et accessible à la raison. Ces principes doivent se lire comme une réplique à Machiavel, une tentative pour moraliser le Prince tout en renforçant son autorité et l'obéissance qui lui est due, sans faire de lui l'unique détenteur d'une «raison d'État» qui échapperait à ses conseillers les plus érudits. Le Prince pour qui Lipse veut écrire accepte encore de se soumettre aux lumières érudites qui lui sont apportées par les humanistes qui l'entourent et qui se proposent d'être ses conseillers. Lipse réécrit le paradigme césarien en complétant le modèle du Prince qui agit, qui s'engage et qui se risque par le modèle du Prince qui écoute, qui délibère et qui consulte.

Alexandre Tarrête

Université de Paris IV-Sorbonne 